Document downloaded from:

http://hdl.handle.net/10251/123538

This paper must be cited as:

Nácher-Soler, VE.; Jurdi, S.; Jaén Martínez, FJ.; García Sanjuan, F. (2019). Exploring visual prompts for communicating directional awareness to kindergarten children. International Journal of Human-Computer Studies. 126:14-25. https://doi.org/10.1016/j.ijhcs.2019.01.003

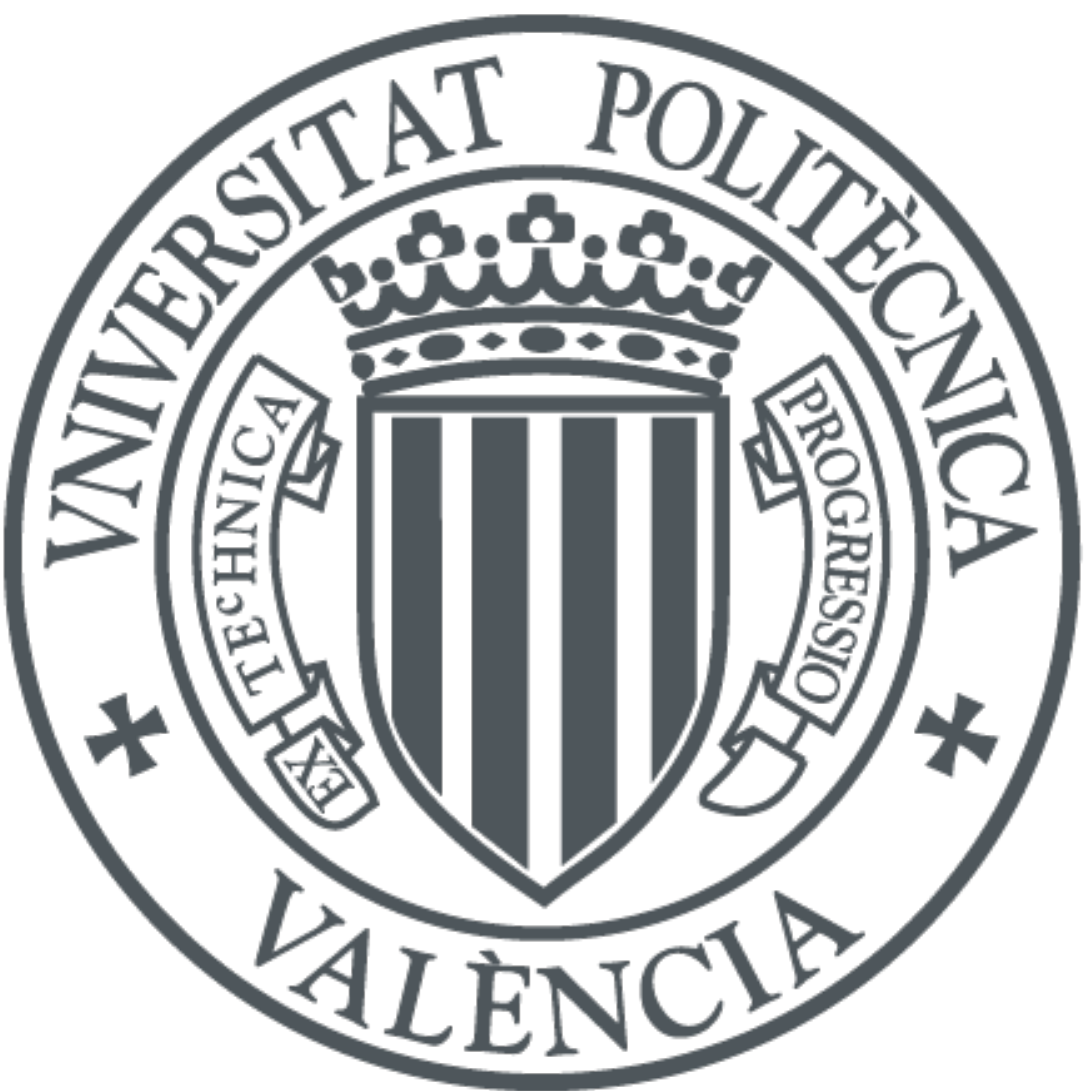

The final publication is available at

http://doi.org/10.1016/j.ijhcs.2019.01.003

Copyright Elsevier

Additional Information 


\title{
Exploring visual prompts for communicating directional awareness to kindergarten children
}

\author{
Vicente Nacher ${ }^{1}$ \\ ISSI Group, Departamento de Sistemas Informáticos y Computación (DSIC), Universitat Politécnica de València \\ Camino de Vera s/n. 46022 Valencia (Spain) \\ vnacher@dsic.upv.es \\ Sandra Jurdi \\ ISSI Group, Departamento de Sistemas Informáticos y Computación (DSIC), Universitat Politécnica de València \\ Camino de Vera s/n. 46022 Valencia (Spain) \\ jurdisandra@gmail.com \\ Javier Jaen
}

ISSI Group, Departamento de Sistemas Informáticos y Computación (DSIC), Universitat Politécnica de València Camino de Vera s/n. 46022 Valencia (Spain)
tel+3496387 7007, fax +34 963877359
fjaen@upv.es

Fernando Garcia-Sanjuan

ISSI Group, Departamento de Sistemas Informáticos y Computación (DSIC), Universitat Politécnica de València Camino de Vera s/n. 46022 Valencia (Spain) fegarcia@dsic.upv.es

${ }^{1}$ Corresponding author 


\section{Exploring visual prompts for communicating directional awareness to kindergarten children}

Abstract. Although a myriad of educational applications using tablets and multi-touch technology for kindergarten children have been developed in the last decade, most of these applications do not fully exploit multi-touch technology since the game world used is limited to the screen only. Considering a larger digital space in tablet-based educational scenarios would be beneficial since it would enable the design of engaging activities driven by curiosity, exploration, discovery and decisions on where the next action is situated in the digital virtual space by directional awareness. This paper therefore investigates kindergarten children's abilities to use a virtual world beyond the screen and evaluates three different types of visual prompts for communicating directional awareness. The results obtained show, firstly, that these specific users are able to use the space beyond the screen boundaries and that the evaluated prompts can effectively communicate information to kindergarten children. The paper also makes a set of recommendations to help designers choose the appropriate type of prompt for their application requirements.

\section{Highlights:}

$>$ H1. Commercial touch apps for kindergarteners only use the screen size as virtual world

$>\quad$ H2. Kindergarteners are able to use the virtual world beyond the screen

$>$ H3. Visual prompts are feasible mechanisms to effectively provide directional awareness

$>$ H4. Several factors must be taken into account when designing visual prompts for children

Keywords. Multi-touch interaction, visual prompts, directional awareness, kindergarten

\section{Introduction}

Since the appearance of touch-based devices such as tabletops and tablets, very young children have had the opportunity to use new forms of interactive media to engage in beneficial educational activities (Nacher et al., 2016). Moreover, as (Hourcade 2007) stated, direct-touch is preferred by children over other mediated pointing devices like the mouse, as it provides a more direct way of selecting options on the screen. Different studies have thus focused on studying 
the suitability of this technology for kindergarten children. The multi-touch interaction has been reported as a more intuitive way of interaction (Smith et al. 2012) that enables users to interact with multi-touch tabletops intuitively (Jokisch et al. 2011); (Mihajlov et al. 2014); (Ioannou et al. 2013). Other studies have evaluated the suitability of smaller devices and reported that even children aged 2 to 3 are able to perform a basic set of multi-touch gestures (tap, scale up, scale down and rotation) on a tablet without assistance (Nacher et al. 2015) and more complex gestures (such as double tap and long press) can be performed when some assistive techniques are used (Nacher et al., 2014). In the same way, Vatuavu et al (Vatavu et al. 2015) showed that children aged 3 to 6 are able to perform touch gestures in both tablets and smartphones. Children's preference for educational tablet-based games has already been demonstrated (Furió et al, 2013) because it involves a natural interaction style requiring little training (FernándezLópez et al, 2013). As a result, several works (Rideout 2011); (Plowman et al. 2012); (Cristia \& Seidl 2015) have pointed out that very young children are frequent users of multi-touch devices and are confronted with this technology even before they fully develop oral communicative functions.

Despite this growth in the use of multi-touch technology by kindergarten children, introducing multi-touch interaction is not the only challenge to be addressed when designing learning applications for them, as discussed in (Falloon 2013). The design process is especially challenging because kindergarteners are in the process of early language development and the younger the children are the more scaffolding of technical nature they need (Neumann 2017), including special communication strategies when using touch screen devices. Application designers thus need adequate strategies to enable young children to interpret information about the applications, such as the gestures to be performed at a given time, the actions needed to go ahead, or information about the spatial location of objects in the virtual world. Therefore, the design of efficient and effective communication visual prompts which gives the user information about the application and the expected actions that the users should make is a key challenge. The design of appropriate semiotics must be addressed since, as pointed out by Derboven (Derboven et al. 2012), multi-touch interfaces can facilitate dialogic learning scenarios in which the dialog is centered around the learning activity itself rather than on the interactions the children are expected to perform each time. Hence, designing visual prompts that avoid the continuous technical scaffolding by adults (i.e. the gestures to be performed, the direction in which a game character should move, etc.) will help caretakers to concentrate on giving cognitive scaffolding (i.e. the learning content to be acquired by the children). In addition, in scenarios in which collaboration is allowed between users, the presence of an appropriate visual prompt understandable for both children and adults would foster a smoother experience and avoid the need to provide incessant instructions and cues. Although designing visual prompts tailored to kindergarten children is not aimed at allowing them to use the 
applications by themselves without adult supervision, the use of appropriate prompts can foster more dialogues about the learning content of the application by reducing the need for technical intervention.

Although the use of touch screen devices by kindergarten children has recently received a great deal of attention in terms of gesture usability, few studies have focused on evaluating suitable semiotics for them. For example, some recent studies (Hiniker et al. 2015) (Nacher et al., 2016) have evaluated possible visual prompts for communicating which are the gestures expected by the application to kindergarten children. These works show that kindergarten children are able to understand semiotic communications when using languages specially designed for them, but not when languages targeted to adults are used. They show that it is possible to design visual prompts for communicating gestures for these specific users and suggest that kindergarteners are able to understand visual cues to communicate information relative to the application. However, although these works point out the importance of designing tailored visual prompts for kindergarteners, there are no studies focused on the assessment of directional awareness communication tailored to this type of user. Designing appropriate directional awareness prompts understandable by children would have several benefits. Firstly, it would reduce the technical scaffolding provided by parents/caretakers when interacting with applications, enabling them to focus on the learning content rather than on the interaction mechanisms. Secondly, it would enable the creation of educational scenarios in which the digital space to be explored exceeds the physical boundaries of the screen, engaging kindergarteners in activities driven by curiosity, exploration, discovery and decision-making about where the next action will take place is in the digital virtual space. Thirdly, it would contribute to the development of this skill (spatial and directional awareness) as an important cognitive ability at this early age.

In this paper we therefore consider the problem of effectively communicating directional clues to kindergarteners and evaluate three different proposed directional awareness visual prompts in order to determine pre-kindergarten users' understanding of the information that they communicate. The three languages under consideration in this work are of a graphical nature because, even though it has already been shown that including instructions in the form of a short text or video clip is suitable for primary school children (Kähkönen \& Ovaska 2006); (Niemi \& Ovaska 2007); (Van Der Meij \& Van Der Meij 2014), pre-kindergarten users do not have the abilities required to read and understand text messages or complex verbal video instructions.

The contributions of this work are manifold: the first is a review of 100 commercial applications of multi-touch devices targeted to kindergarten children, revealing that most of these applications only use the limited screen size as the actual interaction space in which the educational activity takes place. The second contribution is the experimental confirmation that kindergarteners are able to use the space beyond the screen limits as a virtual world. The third is the experimental confirmation that visual prompts can be used to effectively communicate 
spatial directional information to kindergarten children. The fourth contribution is a set of recommendations guiding designers when choosing the language which best fits with the application requirements in terms of time, visual interference and relative positioning awareness.

\section{Related work}

Several studies have evaluated the suitability of multi-touch technology with pre-kindergarten children. However, as has previously been pointed out, there is no standard way of communicating information to children. In order to reduce the number of instructions given by caretakers or parents, several works have evaluated different ways of providing children with instructions about the required interaction. For example, Niemi \&Ovaska (Niemi \& Ovaska 2007), explored an interaction design process with 6 year-old children when instructions are given. Their results show that instructions in the form of animations to show the correct use of complex tools were best understood by children only if spoken instructions were also provided. Obviously, this result could have been expected, because applications for children cannot rely on written text to give instructions due to the lack of reading skills. Another example is the work by Kähkönenet al (Kähkönen \& Ovaska 2006) who explored different ways of providing instructions in applications targeted at 5-6 year-old children with desktop computers. Written instructions and animations were tested and the results showed that despite communicability being especially challenging with young children, following some design guidelines was effective in supporting the communication process. They recommended giving visual cues to trigger children's attention to help them find new content and textual instructions adapted for children and providing video instruction separated from the other modalities so that they can focus on a specific explanation. The results also showed that providing help in the form of audio messages could overcome some of the limitations of written instructions.

McKnight and Fitton (McKnight \& Fitton 2010) performed a test on common touch-screen terminology in which English-speaking children aged between 6 and 7 were asked to perform a basic set of touch gestures from audio and written instructions. Their results showed that these young users had little or no trouble in understanding most of the instructions and completed the task easily. Giving instructions in both textual and audio form was thus seen to be useful, and even less familiar terms such as "slide" or "swipe" were well understood. However, they concluded that due to the children's different abilities it was hard to establish a consistent link between a term and a touch gesture, which makes giving instructions particularly critical in systems designed for them due to their limited vocabulary and reading skills.

Another approach to communicating multi-touch gestures to 5-6-year-olds was explored by Baloian et al. (Baloian et al. 2013). The study used words and pre-recorded audio sources to ask users to identify and perform different gestures. Instead of referring to gestures as adults would 
(e.g. by their name), the researchers used metaphors for each of the application's gestures. They associated each gesture to a specific "recallable" character (e.g. a jumping grasshopper for a double tap, a walking ladybug for a drag gesture or a hovering butterfly for a tilting gesture). However, the results showed no strong correlations between performance and the behavior of the characters that the children liked or disliked the most.

The cited works considered giving instructions with a combination of text, audio and/or visual cues with children aged 5 to 7 , showing that they are ready to use this type of communication despite its having some minor issues. However, applications for very young children cannot rely on written text to give information due to the lack of reading skills and there is no standardized way to name touch gestures, so using audio cues may present several issues and may cause interferences in the parents/caretakers-children dialogues.

Hiniker et al (Hiniker et al. 2015) evaluated prompts such as in-app audio, on-screen demonstrations (with hand demos or changing the visual state of the item) and instructions by an adult model for eliciting gestures such as double tap, horizontal and vertical swipe and shaking the tablet with children aged between 2 and 5 . Their results showed that although the most effective technique was adult guidance, children aged 3 years or older were able to follow other types of cues. Nacher et al (Nacher et al., 2014) (Nacher et al. 2017) analyzed the communicability of three types of touch gestures (in-place, one-contact dynamic \& two-contact points dynamic gestures) comparing three types of visual prompts with children aged 2 to 3 . Their results showed that although the iconic approach designed for adults is not appropriate for young children, the two animated languages evaluated had high success rates (reaching 90\%) when communicating gestures which involve movement (drag, rotation \& scales). Hence, the basic reasoning related to the interpretation of moving elements on a surface can be effectively performed during early childhood. This is an interesting result because developmental psychologists such as Levine and Piaget suggest that kids develop spatial reasoning during middle childhood (Levine et al. 1999) (Piaget 1973). However, the above-cited study suggests that basic reasoning related to the interpretation of moving elements on a surface can be effectively performed during early childhood.

These latest works focus on evaluating semiotics for giving instructions which use only visual cues with children aged from 3 to 5 . Although their results showed that these approaches are feasible and understandable for these users, the studies focus on semiotics for giving instructions to children but not for giving any type of application information, such as directional awareness, spatial information, application goals, etc. As Markopoulos and Bekker (Markopoulos \& Bekker 2003) point out, interfaces for children should be designed according to their development stages and their actual needs.

Despite the lack of works that consider communication strategies for kindergarterners with respect to spatial directional awareness in the context of computer-based learning applications, 
there have been a number of works that propose strategies for teachers to help children develop spatial literacy. This is the case of Golbeck (Golbeck 2005), who proposes ways of promoting spatial literacy in the study of mathematics, social studies, science, literacy, and visual arts. Interestingly, the author affirms that "with experience and growing memory capabilities, children shift from a uni-axial to a bi-axial system for spatial representation". This would encourage the use of current interactive technologies to help children make this shift by exposing them to experiences in which spatial awareness is exercised. It is also interesting to point out here that many works (Case et al. 2008; Demetriou et al. 2002; Case et al. 2001) have studied how children develop spatial thinking by analyzing how they produce drawings and how space is represented in them, e.g., from depicting objects floating in space weightlessly without a reference coordinate system in their pre-axial phase to fully integrating two or more reference lines and considering perspective and depth relationships by age 10. However, these studies just consider what spatial information children are able to produce in terms of drawings but not whether they are capable of decoding, understanding and using spatial knowledge that is provided to them by some sort of graphical language. These studies, which conclude that children are not able to consistently create bi-axial representations until the age of 8 have probably made designers of learning applications for kindergarteners think that at this early age children are not able to interpret and use bi-axial spatial or directional information.

The present study will therefore evaluate whether this assumption is sound or whether there is space for improvement by designing applications that help children to explore bi-axial interactive spaces whose limits are outside the reduced screen size of current multi-touch devices.

\subsection{Industrial perspective on the communicability of spatial and directional awareness in touch devices}

Besides the review of the studies from a research perspective in the previous section, it is important to examine how commercial applications are addressing the communicability of spatial and directional information about their digital world. With this purpose, 100 educational applications $^{2}$ were randomly selected from the collection of the kindergarten educational applications in the Android App Store and were analyzed in terms of usage of a space beyond what is being displayed on screen (i.e. whether the application camera is fixed and always displays the whole space or can be moved to reach other parts not displayed at a given time). When there was a digital world beyond the boundaries of the screen, we analyzed the mechanisms used to provide directional awareness to users, if any.

${ }^{2}$ goo.gl/vG88fM 
The results of this analysis revealed that only $33 \%$ of the applications used a game world beyond the screen size. Most of the applications targeted to kindergarten children are limited to simply using the screen size as the digital world that is always shown completely. Only $5 \%$ of the reviewed applications (15.15\% of the applications that use the world beyond the screen) use any type of mechanism to provide directional awareness to the users.

Two different techniques were identified: the first (used in three applications) is an arrow to signal the direction that the character has to follow to proceed in the task/game (see Figure 1).

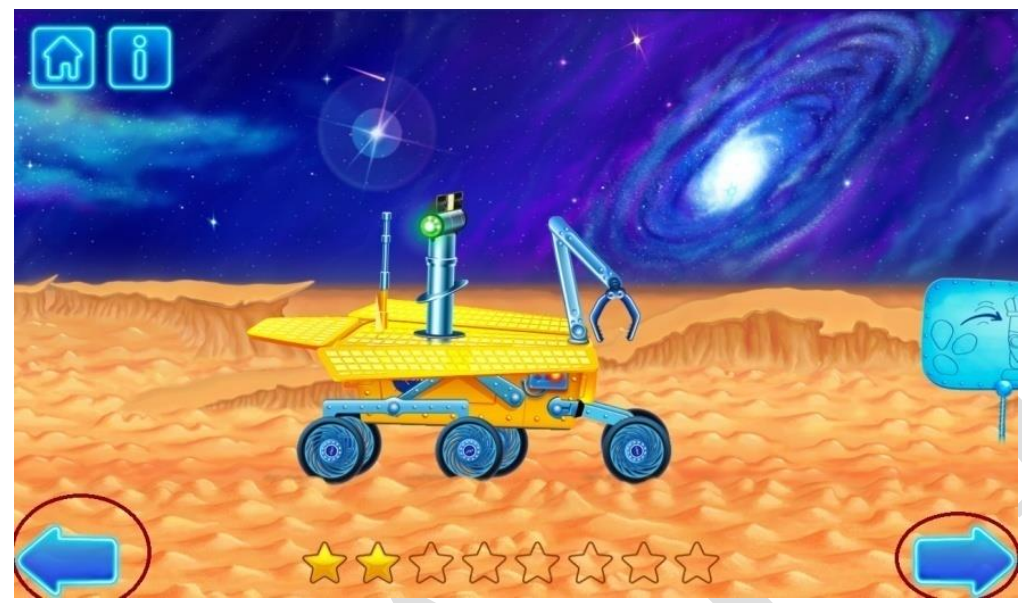

Figure 1. Example of communication used in a commercial application (Space Mission developed by Thematica - educational and fun apps for kids).

The other technique (used in two applications) is a dynamic semiotic that indicates the direction in which the target objects (i.e. the objects to be reached by the main character of the game) can be found when they are not being displayed on the screen. To do that, when an object "disappears" because its position is outside of the screen limits, a miniature of this object is shown in the last visible position and a dynamic arrow moving around it indicates the direction in which the object can be found (see Figure 2).

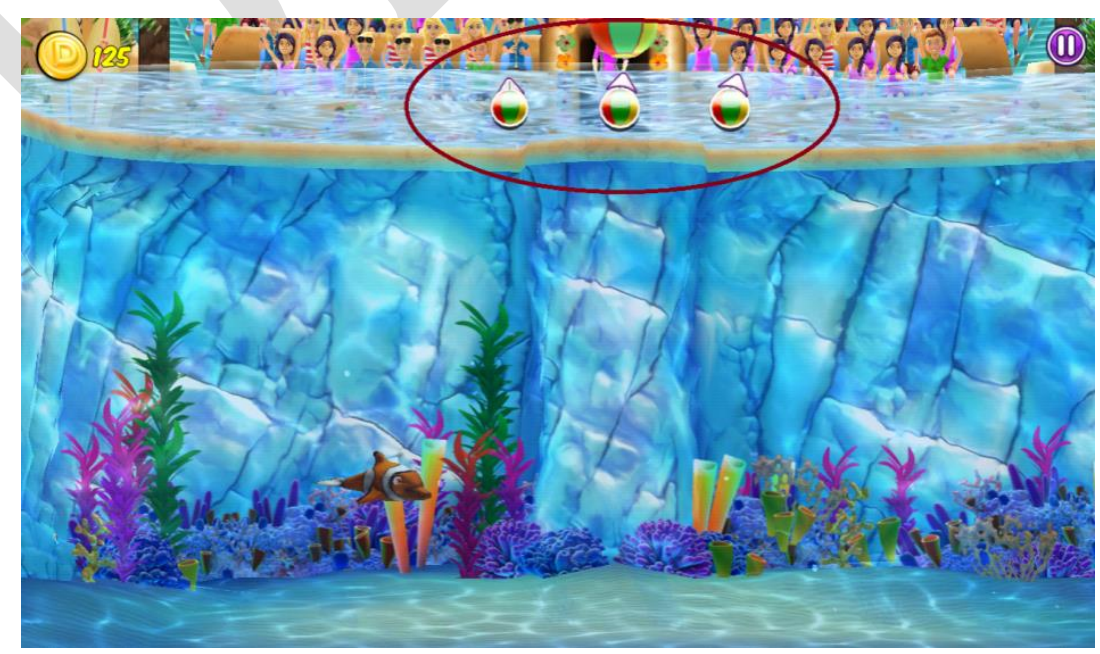

Figure 2. Example of communication used in a commercial application (My dolphin show developed by Spil Games). 
The main conclusion that can be drawn from these results is that, even though there is a popular commercial trend in developing apps for kindergarten children with touch-screen technology (Nacher et al. 2015), developers in general simply use the screen size as the boundary of the game/application world. Few applications use some sort of prompts to provide directional or spatial awareness to users and there is no standard and validated way of providing this information. In this work we therefore evaluate whether kindergarten children are able to interact/play with applications in which the digital world is not limited to the screen only and then evaluate three different types of visual prompts to provide spatial directional feedback to children when interacting with an application of this type. The empirical evaluation and the results obtained in this work are a step forward in the process of obtaining an effective language to communicate directional information to kindergarten children that could be used in educational applications based on this technology.

\section{Visual prompts for directional awareness}

In this work, we use visual prompts to provide kindergarten children with directional awareness of the digital world beyond the area being displayed on the screen at a specific time. This directional awareness is the information about the direction that the main character in the game should follow to reach the different objects that need to be picked up or visited in order to succeed in the game task. In this context, the selected game style is that of adventure games in which children have to control the movements of a character to explore the surrounding space in search of items. The choice of this type of game style is because it stimulates curiosity (Collins \& Stevens 1981) (Malone 1981) and can potentially facilitate a range of different learning styles such as tutoring, practice and self-learning (Dempsey et al. 1994). In addition, this type of games fosters learning discovery which is a technique that helps learners to create and organize their knowledge, since they draw upon past knowledge and experience to infer underlying strategies and gain understanding of concepts (Honomichl \& Chen 2012). Knowledge discovery is also beneficial for students' motivation, since those who discover information for themselves are more motivated to achieve educational goals and more likely to remember the information learned (Bruner 1960). Hence, the proposed directional awareness languages need to give information about where the main character of the game is with respect to the target object in order to help children to effectively guide the character towards the correct destination. In order to provide informational awareness, the science of semiotics (Bradbury, 1978) has proposed the use of signs. A sign is anything that creates meaning, anything that can be used to represent something else. As Peirce pointed out (Peirce, 1902), the form a sign takes, it's signifier, can be classified as one of three types: an icon, an index or a symbol. An icon has a physical resemblance to the signified, the thing being represented. An index shows evidence of what's 
being represented. Finally, a symbol has no resemblance between the signifier and the signified. Previous works regarding the use of visual prompts to communicate information to children (Downs et al. 1988) (Thomas et al. 1994) (Leekam et al. 2008) reveal that to understand symbols, children require an understanding of the object-referent relation and the informational value of the sign along with its substitutional function. If the child can neither detect the information a sign conveys nor use it as a representation of its referent, a lack of communication is the consequence. As pointed out by (Catling 2005), it is true that the ability to derive proper meaning from symbols on maps is developmentally related and gradually improved, (Goria \& Papadopoulou 2012) maintain that preschoolers use iconicity to a large extent instead of symbols to represent spatial cartographic information. This would suggest that, in theory, an iconic style would be a good candidate to communicate directional awareness. However, there are no studies that confirm this superiority with respect to a symbolic choice when communicating directional awareness. When communicating this awareness, icons need to be placed within a cartographic context to convey direction and this issue has not been previously studied in preschoolers. In this study we therefore selected three visual styles to study their effectiveness with respect to directional awareness: a pure symbolic language, an iconic with local cartography language and an iconic with global cartography language. In order to select suitable candidate languages for evaluation, several workshops took place with kindergarten educators and pedagogical experts. As a result, the following mechanisms were selected for evaluation with these children:

- Mini-map (icons over global cartography): in this case, a miniature map is placed at the bottom right corner of the screen (see Figure 3). This map contains the entire digital world displaying all the existing objects and the main character. In this case, the destination element is marked in the mini-map with a red stripe around it (see Figure 3).Using this technique, the users are aware of the position of all the objects in the game and the distance to reach them. This type of language is often used in games targeted at adult players. Mini-maps could be designed to contain not only icons but also symbols and in an indexed way. In our approach we have decided to make use of iconic signs based on previous studies revealing the use of iconicity by children (Goria \& Papadopoulou 2012). 


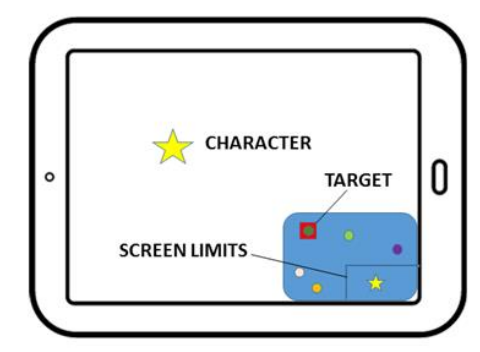

(a)

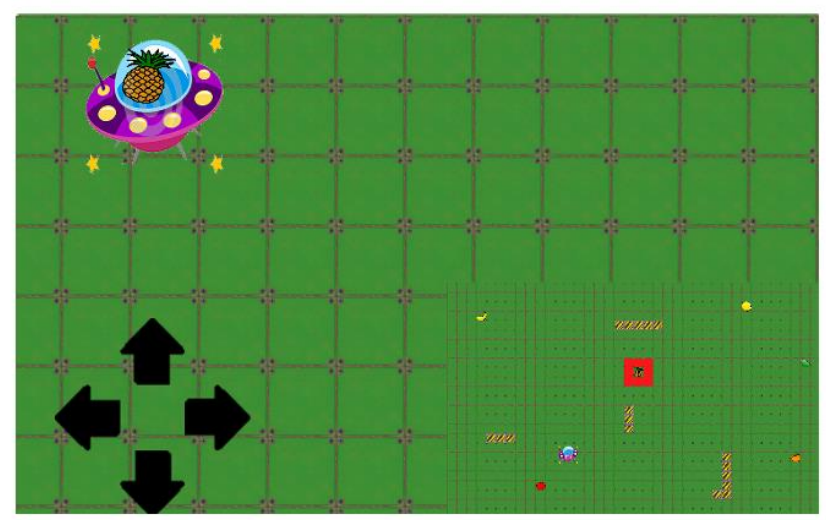

(b)

Figure 3. Example of the task with the mini-map (a) abstraction (b) application snapshot cropped and enlarged in the mini-map zone.

- Border-Floating thumbnails (icons over local cartography): in this technique, miniatures of the objects that are not visible on the surface appear at the border of the screen. The position where the miniature is shown is the intersection between the vector that links the character to the corresponding object and the screen border (see Figure 4). The miniature positions are dynamically updated according to the relative character's position at a given time. With this technique, the language only represents the objects that are outside the current screen display.

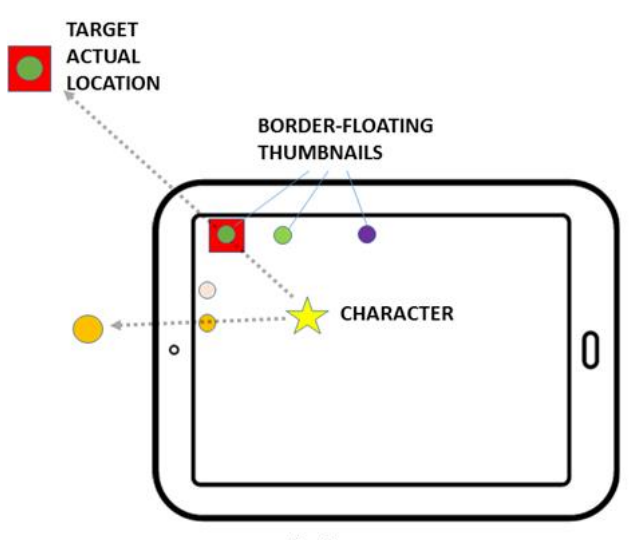

(a)

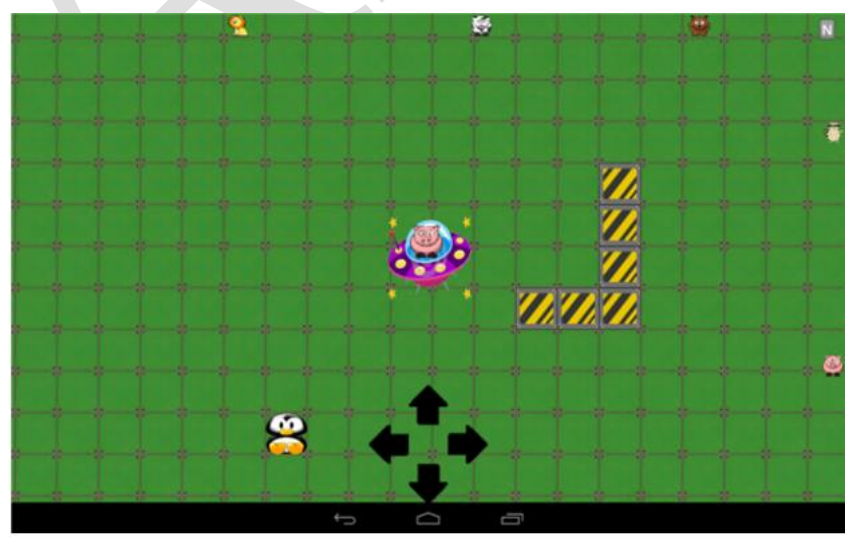

(b)

Figure 4. Example of the task with the thumbnail language (a) abstraction (b) application snapshot.

- Arrow (symbolic): in this case, the technique uses an arrow to indicate the direction to follow to reach the current target (see Figure 5). The arrow is dynamically updated and will move around the character according to the trajectory to be followed to reach the destination. In this technique, information on the relative positions of other objects is not represented. 


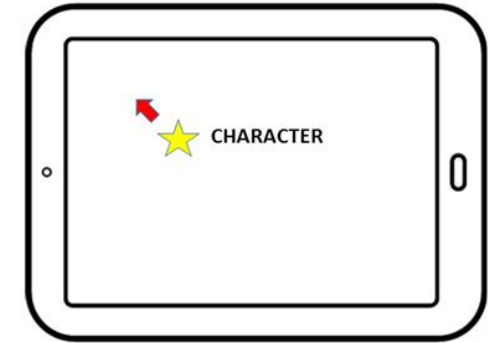

(a)

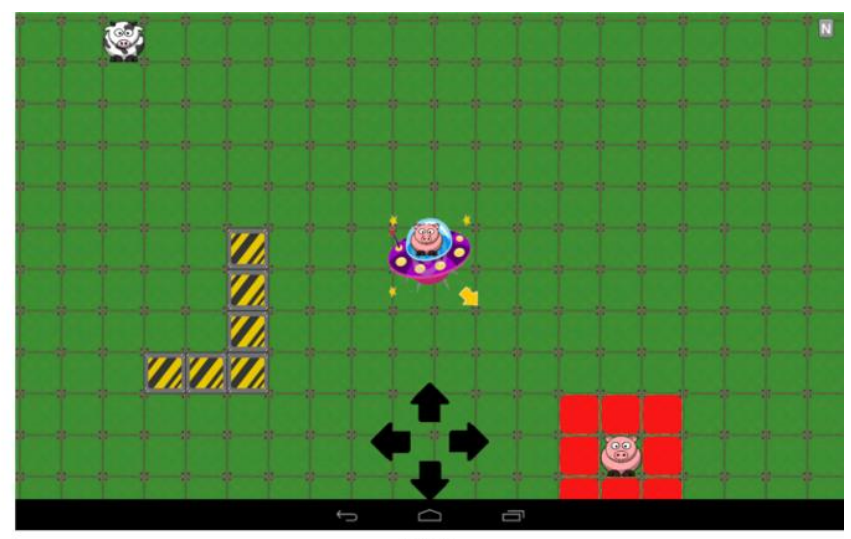

(b)

Figure 5. Example of the task with the arrow language (a) abstraction (b) application snapshot.

\section{Study context}

The overall goal of our study was to test the suitability of the previous visual prompts for providing directional awareness of the objects in a digital 2D game world to kindergarten children and to evaluate their effectiveness and efficiency. One of the mechanisms, the minimap, is often used with adult users and the other two were designed for children. Hence, using the GQM (Goal Question Metric) template (Basili et al. 1994), our goal can be defined as follows: analyze three different visual prompts for the purpose of evaluating their suitability from the viewpoint of effectiveness and efficiency in the context of providing directional awareness of the objects in a digital game world to kindergarten children.

For this study we considered children of both genders aged between 4 and 7. According to developmental theories, children are continuously developing and refining their cognitive skills and are in the preoperational stage of development until the age of 7 (Piaget 1973). As we were interested in exploring how the proposed visual prompts are performed by young children and how the development of their cognitive skills affects their proficiency, we defined three age groups; children aged between 4-5, 5-6 and 6-7.

Consequently, the research questions of this work may be formulated as follows. The first research question is about the appropriateness of using visual prompts to communicate directional awareness:

- RQ1: Is any of the considered visual prompt effective in providing directional awareness to kindergarten children?

Then, four research questions are stated and will be answered for each factor $F_{i}$ considered (where $i=$ Type of visual prompt, Age and Gender)

- RQ2: Is the effectiveness in the task affected by the factor $\mathrm{F}_{\mathrm{i}}$ ?

- RQ3: Is the efficiency of the task affected by the factor $F_{i}$ ?

- RQ4: Is the relative positioning awareness in the task affected by the factor $F_{i}$ ?

- RQ5: Is the level of visual interference with the task affected by the factor $\mathrm{F}_{\mathrm{i}}$ ? 


\subsection{Participants}

Sixty children aged from 4 to 7 years old took part in the experiment (Mean $(M)=67.4$ (months), Standard Deviation $(\mathrm{SD})=9.75)$ with a gender distribution of 26 males and 34 females.

The children were split up into three balanced age groups, i.e. they were grouped by age, with each age group a comprising the ages in $[a+1[$. The distribution of the age groups is shown in Table 1.

Table 1 . Number of participants by age group

\begin{tabular}{|l|l|}
\hline Age group & \#Participants \\
\hline 4 & 20 \\
\hline 5 & 20 \\
\hline 6 & 20 \\
\hline
\end{tabular}

The 4 to 7 year age range was chosen in order to explore how the proposed prompts are understood and learned by children in the earliest stage of development. The youngest users were children aged 4 years because in previous studies (Nacher, Ferreira, et al. 2016), children were found not to be able to move/guide a character in a $2 \mathrm{D}$ world using indirect drag techniques with acceptable success until 4 or older. Parental authorization was obtained before carrying out the study.

\subsection{Equipment}

The interaction framework for the experiment was implemented in Java using the LibGDX framework. The devices used for the experiment were BQ Edison 3 tablets with Android 4.4. The tablets were equipped with capacitive multi-touch screens.

\subsection{Task}

We wanted to design a task to test several factors and to ensure that all the users have the same conditions in order to compare the results. The task would allow the suitability of using the virtual space "beyond the screen" limits to be assessed with kindergarteners, evaluating whether they are able to reach several targets in a virtual 2D world and determine the suitability of the designed visual prompts to facilitate dialogic learning scenarios in which the dialog is centered on the learning activity itself rather than on the interactions the children are expected to perform each time. This task was chosen because it is an initial step in designing game scenarios in which discovery and curiosity can be fully fostered to support storytelling activities for a bidimensional virtual space, adventure games in an open space, etc.

Therefore, the task consists of a game in which a spacecraft (see Figure 7) has to travel within a digital 2D space in order to pick up, in a predefined order, three of the six objects scattered 
around the digital world (see Figure 6 for examples (two examples by topic) of the objects scattered in each topic), which includes some blocks that cannot be traversed, to force children to plan trajectories that avoid crashing into them.

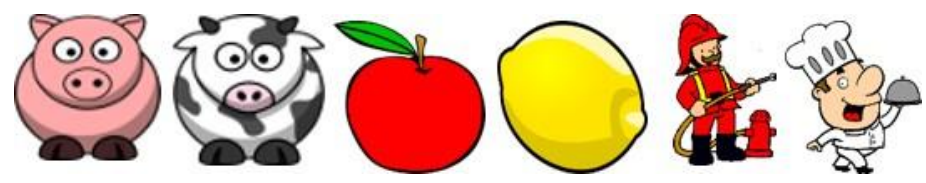

Figure 6. Example of some objects of the three different topics to pick up in the game (animals, fruits and jobs).

The interaction technique selected to move the spacecraft was button-based dragging. This technique consists of four arrow-shaped buttons that are used to move the target object in the four basic directions (i.e. up, down, left and right). The buttons were placed at the bottom-center of the screen (see Figure 7). With this technique, users can move the target by tapping and holding one finger on the button that symbolizes the desired direction. This mediated interaction mechanism has been evaluated in previous studies (Nacher, Ferreira, et al., 2016) and shown to be suitable for children aged 4 years and older. This study also revealed that it was the best indirect drag technique of all those evaluated if the main priority is to avoid undesired collisions with objects in the game.

Using the button-based dragging technique, children have to guide the spacecraft through the digital world in order to pick up the three proposed objects. The current target to be picked up at a given time appears on the spacecraft (see Figure 7) and the cells around it are colored in red. The children then have to use the corresponding visual prompt to obtain directional awareness and reach the target. When the correct object has been picked, the next one appears on the spacecraft and when the three objects have been picked up the task is over.

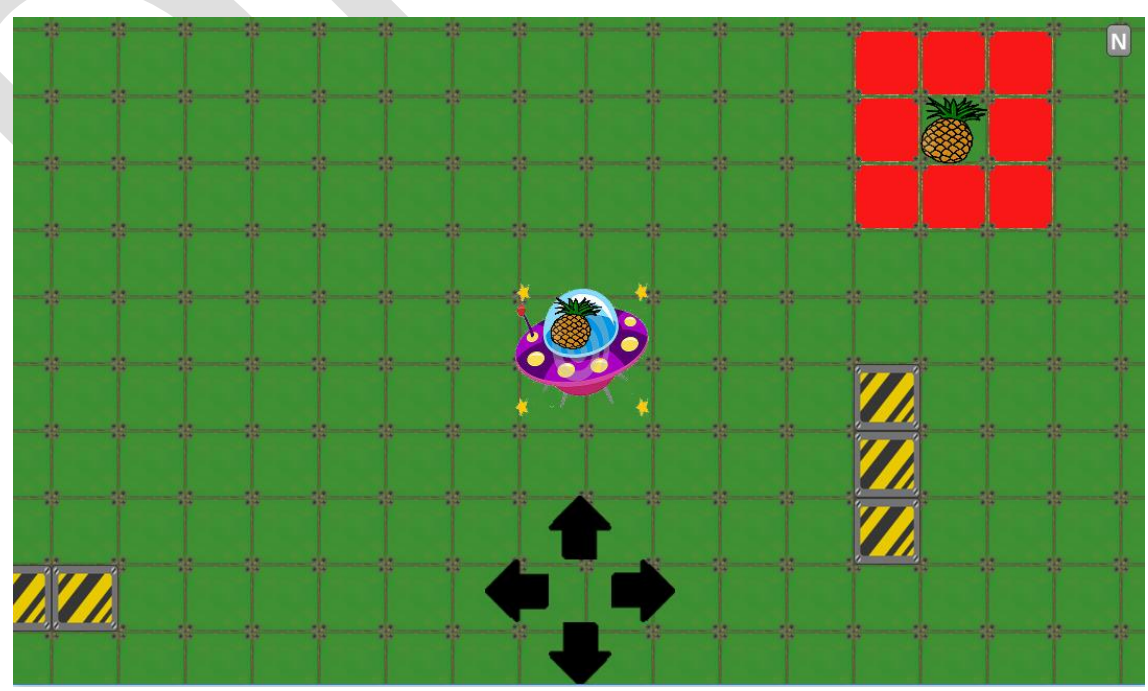

Figure 7. Task example without visual prompt. Topic: Fruits.

There were three topics for the task: animals, fruits and jobs. In each topic, the pickup order is predefined and the same for all children in order to compare the results. 


\subsection{Procedure}

The experiment was carried out on three consecutive days (one technique each day). The children performed three repetitions (one per topic) of the task daily, using one of the visual prompts described above. The order in which the topics were presented and the order in which the languages were evaluated each day were randomized per subject to avoid learning effects.

At the beginning of each session, the children participate in a 5-minute learning phase with an instructor in which children get acquainted with the task and the interaction technique using the buttons-based drag. In this learning phase the instructor teaches the children that there are several objects scattered around the game world that they have to pick up in a specific order. The children are told that the spacecraft is the main character to be controlled and it displays the current object to be picked up. After these introductory explanations, the instructor makes sure that none of the children fails to identify themselves with the spacecraft. During the training session there is no directional awareness language displayed. When the test begins the children have to perform three repetitions of the task (one per topic) with the assigned visual prompt and there is no additional external adult intervention. When a correct object is picked up, the platform gives a positive audiovisual feedback. In the same way, if the object picked up is not the correct one a negative audiovisual feedback is given by the platform. If the instructor observes that the participant is not able to find or pick up an object in a given time, it is marked as undone and the child continues with the next item. For each task, the system records the following information: the completion time to pick up the objects; the success rate (whether they pick up the correct object in a given trial); any collisions with the blocks on the surface, the number of incorrect pickups and the distance in pixels travelled by the spacecraft across the game world in order to compare it with the optimal path for the task. A qualitative analysis is also carried out from the notes taken by an external observer during the experimental sessions. To sum up, the children participate in the experiment for three days and each day they perform three repetitions of the task (one per topic) to pick up three of the six scattered objects in each topic in the game using a different type of visual prompt each day.

\subsection{Design}

Six dependent variables were defined: success rate, completion time, travelled path, changes of direction, collisions with blocks and incorrect pickups. A mixed design was used as all the participants tested the three types of directional awareness visual prompts. A repeated measures ANOVA (with an $\alpha=0.05$ ) was carried out with the within-subject factor Visual prompt with three levels (Minimap vs. Thumbnails vs. Arrow) and the between-subject factors age group (4 years vs. 5 years vs. 6 years) and gender (Male vs. Female). A Mauchly's Test of Sphericity (Mauchly 1940) was carried out in order to determine whether the data met the sphericity assumptions. The tasks that did not comply with these assumptions were applied a Greenhouse- 
Geisser (Abdi 2010) correction and were reported in the results section with F-values with fractional degrees of freedom.

\section{Results}

\subsection{Success rate}

In order to aggregate the success variable over the three repetitions of the task with each language, the variable was expressed as a percentage according to the number of repetitions performed successfully. The success rate of each visual prompt is given in Table 2 and shown graphically in Figure 8.

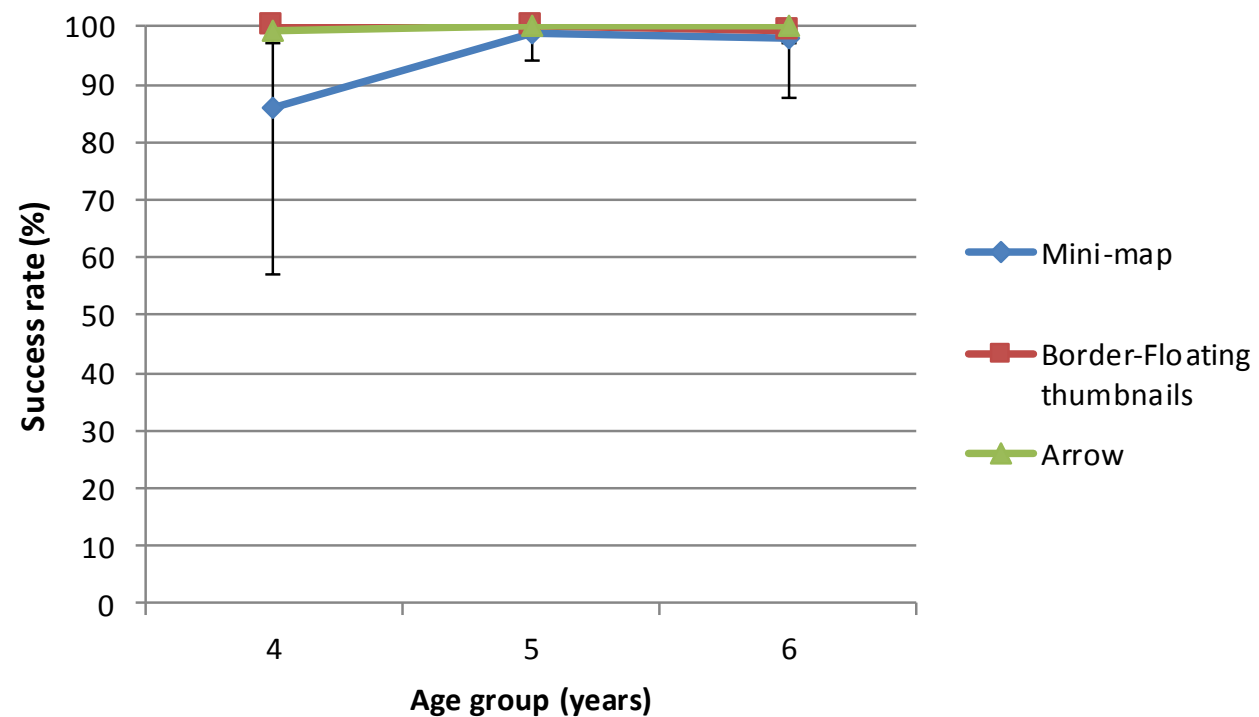

Figure 8. Success rate by visual prompt and age group.

The repeated measures ANOVA revealed significant differences in the within-subject factor visual prompt $[\mathrm{F}(1.019,58.074)=5.621, \mathrm{p}=.021]$ and in the between-subject factor Age group $[\mathrm{F}(2,57)=3.298, \mathrm{p}=.044]$ but not in the between-subject factor Gender $[\mathrm{F}(1,58)=.261, \mathrm{p}=$ .611]. The post-hoc tests (see Table 8) revealed significant differences between the mini-map approach and the other two techniques. As can be seen in the figure, the difference in the success rate comes from the younger age group having a lower success rate. Moreover, the ANOVA revealed that there are no significant main effects visual prompt*age group interaction $[F(2.038,58.074)=2.979, p=.058]$. Hence, success rate evolves with age in the same way in the three visual prompts.

\subsection{Completion time}

With the purpose of evaluating the completion time spent by each user to perform the task, the average of each subject's successful task was used. The unsuccessful tests were excluded in the completion time analysis. Completion time can be seen in Table 3 and Figure 9 by visual prompt and age group. 


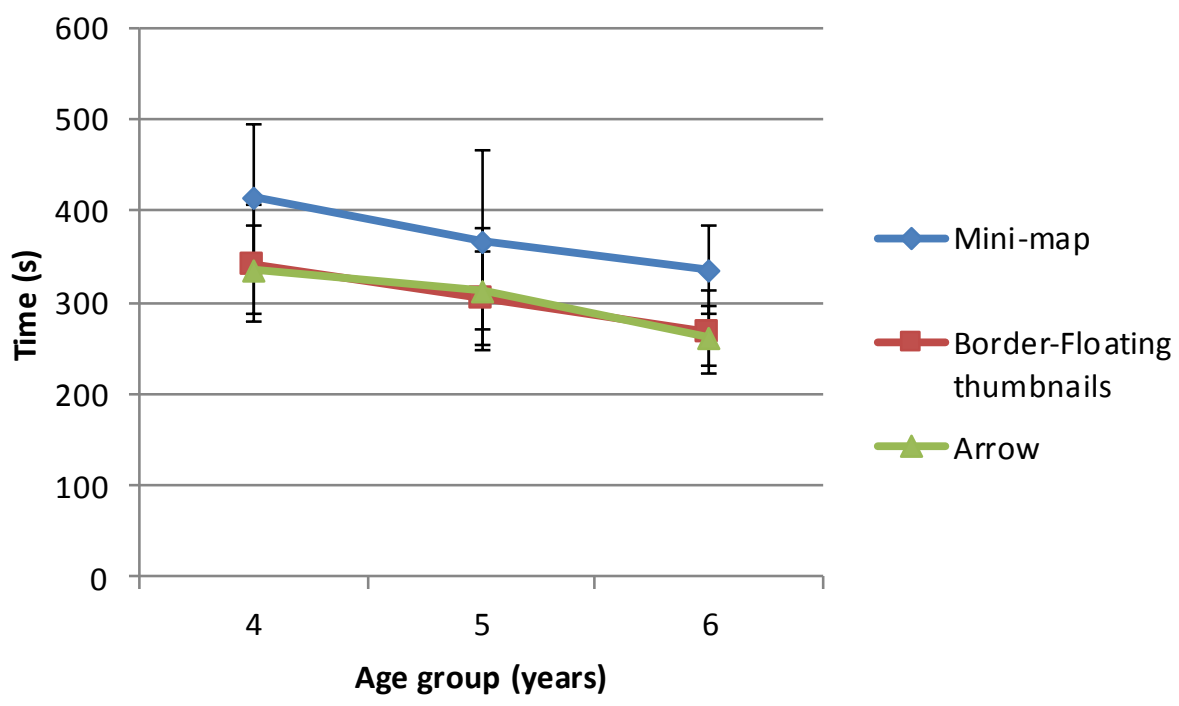

Figure 9. Completion time by visual prompt and age group.

The repeated measures ANOVA revealed significant differences in the within-subject factor visual prompt $[\mathrm{F}(2,98)=36.927, \mathrm{p}<.001]$ and in the between-subject factor Age group $[\mathrm{F}(2,49)$ $=9.954, \mathrm{p}<.001]$ but not in the between-subject factor Gender $[\mathrm{F}(1,50)=.808, \mathrm{p}=.373]$. The conducted post-hoc tests (see Table 8) revealed that there are differences in the completion time between the mini-map language and the other two techniques, the mini-map being significantly slower (22.4\% more time needed to complete the task) than the others. The post-hoc tests (see Table 8) conducted on the age group factor revealed that there are differences between all the age groups in terms of completion time; the older they are the faster they perform the task.

The ANOVA also revealed that the completion time evolves with age in the same way for the three languages, since there are no significant effects with the visual prompt*age group interaction $[\mathrm{F}(4,98)=.373, \mathrm{p}=.827]$.

\subsection{Relative positioning awareness}

When providing location awareness, a key issue is the ability of the prompts system to effectively communicate the relative position of a target destination with respect to the current position of the user. In order to assess the effectiveness of the languages under evaluation we measured the optimality of the traversed path to reach a given destination and the number of changes of direction required to reach the target. These are two indirect metrics that provide a quantitative measure of the cognitive effort that pre-kindergarteners have to make to understand where the final destination is with respect to their current position.

\subsubsection{Optimality of traversed path}

In order to evaluate the optimality of the traversed path by the subjects with the main game character, the distance in pixels that the main character travels in each test was gathered $\left(\mathrm{d}_{\text {travelled }}\right)$. This distance was compared to the distance that would have been travelled had the 
path been optimal $\left(\mathrm{d}_{\text {optimal }}\right)$, i.e. the shortest path to pick up all the targets, and the absolute error was calculated. This error is expressed as a percentage over the total path distance (100 $\left.\cdot \mid \mathrm{d}_{\text {travelled }}-\mathrm{d}_{\text {optimal }} / / \mathrm{d}_{\text {optimal }}\right)$ by visual prompt and age in Table 4 and Figure 10.

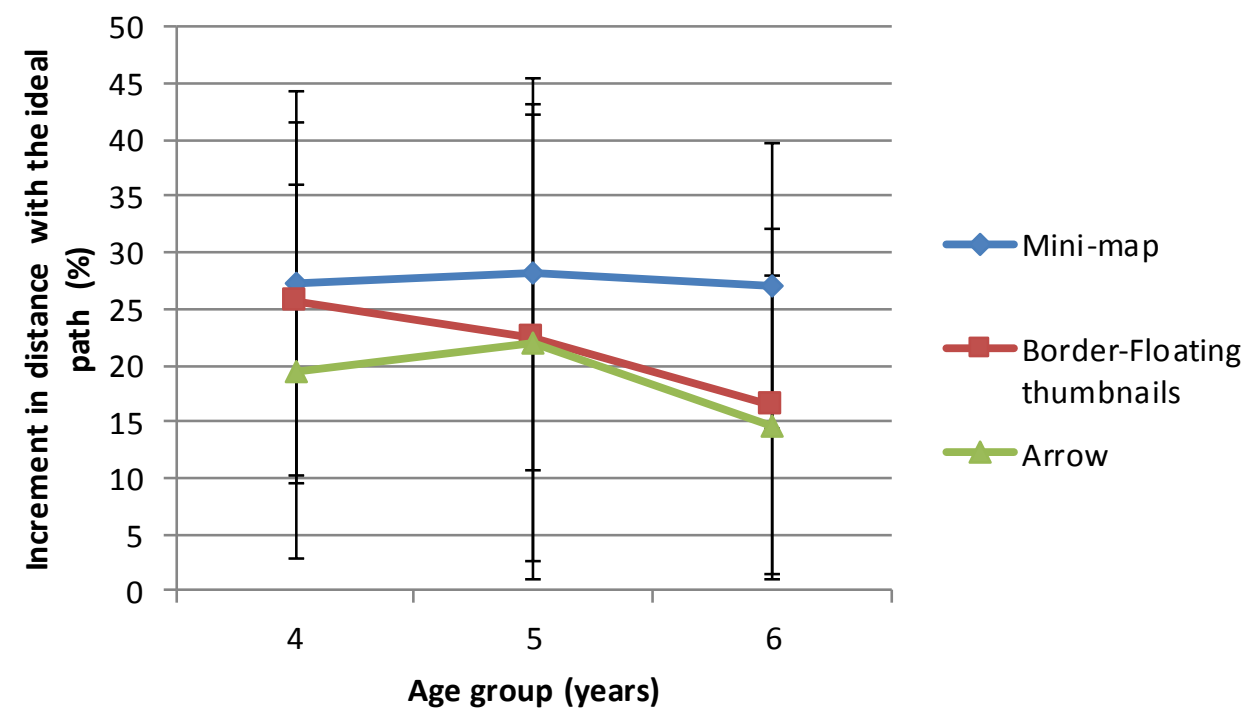

Figure 10. Increment of distance travelled by visual prompt and age group.

The repeated measures ANOVA revealed significant differences in the within-subject factor visual prompt $[\mathrm{F}(2,106)=5.700, \mathrm{p}=.004]$. The conducted post-hoc tests (see Table 8 ) revealed that there are differences in the increment of the travelled path between the mini-map and the arrow technique because users travelled a significant longer path when using the mini-map, $\left(\mathrm{M}_{\text {mini-map }}=27.48\right.$ and $\left.\mathrm{M}_{\text {arrow }}=18.71\right)$. No other significant differences were revealed by the post-hoc tests. The analysis did not reveal any significant differences in the between-subject factor Age group $[\mathrm{F}(2,53)=1.187, \mathrm{p}=.313]$ and the between-subject factor Gender $[\mathrm{F}(1,54)=$ $.610, p=.438]$. Hence, the age and gender did not have a significant impact in the increment of distance in the travelled path.

The ANOVA also revealed that the increment in the travelled path evolves with age in the same way for the three languages, since there are no significant effects with the visual prompt*age group interaction $[\mathrm{F}(4,106)=.602, \mathrm{p}=.662]$.

\subsubsection{Changes of direction}

Another way of evaluating the relative position awareness during the task is to measure the number of times each child changed the direction of the movement of the main character. These changes of movement direction reveal that the user is changing his (her) mind about the path to be followed and indirectly measure the effectiveness of the visual prompt to communicate the correct direction to be followed. The number of times that children changed the direction in a task is given in Table 5 and shown graphically in Figure 11 by visual prompt and age group. 


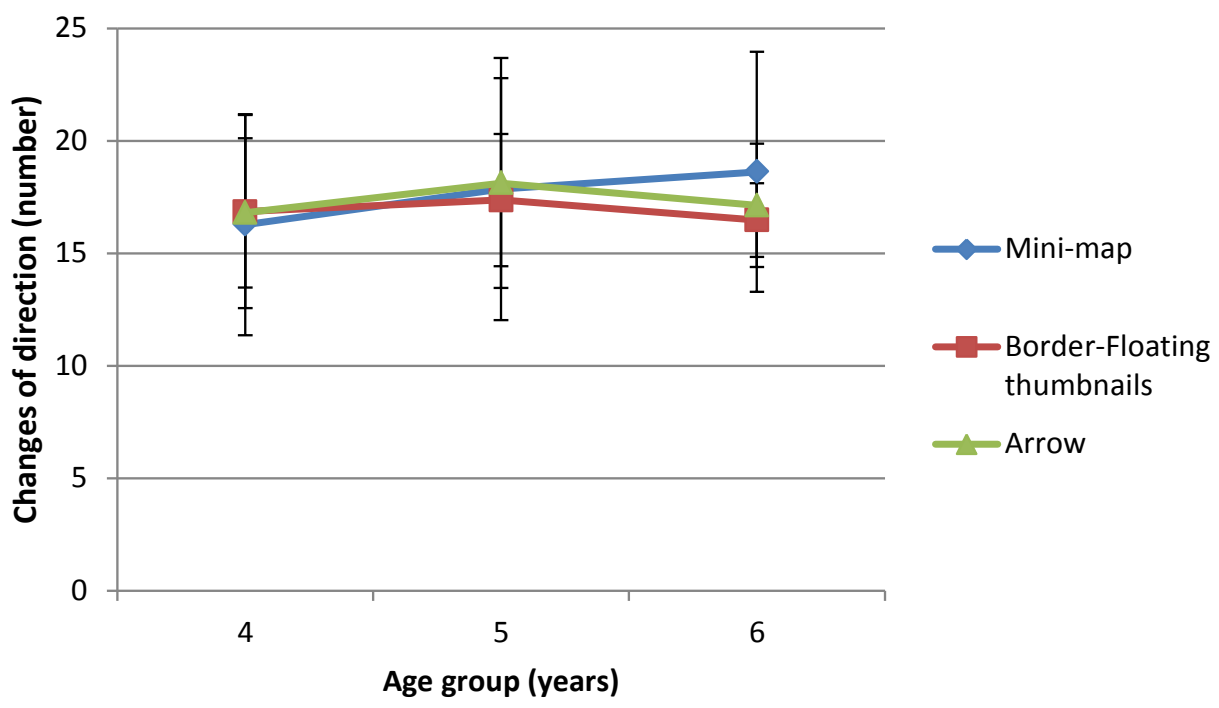

Figure 11. Changes of direction by age group and visual prompt.

The repeated measures ANOVA did not reveal significant differences in the within-subject factor visual prompt $[\mathrm{F}(2,114)=.496, \mathrm{p}=.610]$ or in the between-subject factors Age group $[\mathrm{F}(2,57)=.865, \mathrm{p}=.427]$ and Gender $[\mathrm{F}(1,58)=.000, \mathrm{p}=.987]$.

It also revealed that the number of changes of direction evolves with age in the same way for the three techniques since there are no significant effects with the visual prompt*age group interaction $[\mathrm{F}(4,114)=.756, \mathrm{p}=.556]$.

\section{$5.4 \quad$ Visual Interference}

\subsubsection{Collisions with blocks}

Data was gathered on collisions with the blocks in order to evaluate the degree of interference of the visual prompt with the task at hand by measuring the ability of children to avoid obstacles when guiding a character in a 2D world. The number of collisions of the character in a task can be seen in Table 6 and Figure 12 by visual prompt and age group. 


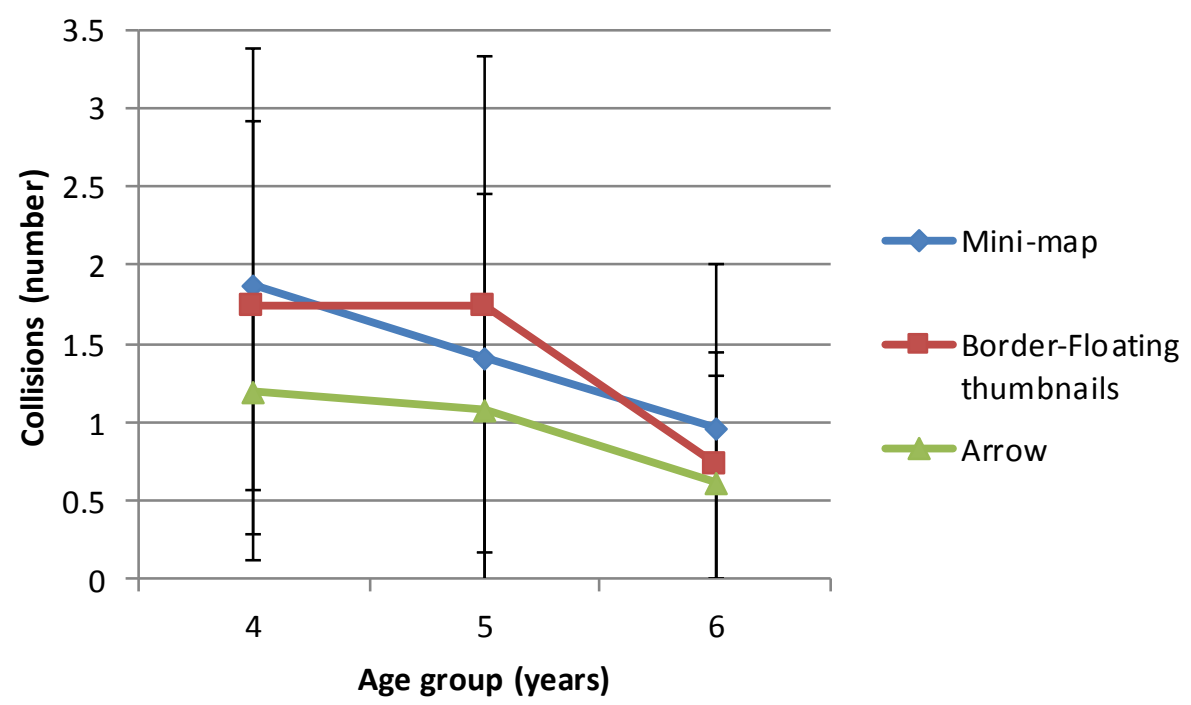

Figure 12. Number of collisions by visual prompt and age group.

The repeated measures ANOVA revealed significant differences in the within-subject factor visual prompt $[\mathrm{F}(2,114)=14.600, \mathrm{p}<.001]$ and in the between-subject factor Age group $[\mathrm{F}(2,57)=4.174, \mathrm{p}=.020]$ but not in the between-subject factor Gender $[\mathrm{F}(1,58)=.245, \mathrm{p}=$ .623].The conducted post-hoc tests (see Table 8 ) revealed that there are significant differences in the number of collisions with blocks between all the types of visual prompts being the mini-map technique in which children had a higher number of collisions, followed by the border-floating thumbnails and, finally, the best technique to avoid collisions with blocks according to the results was the arrow. In addition, although there is a trend to reduce the number of collisions with age (Figure 12) the conducted post-hoc tests (see Table 8) with the age group factor only revealed significant differences between the 4-year-old and 6-year-old children, the older ones having the lowest number of collisions.

The analysis also revealed that the number of collisions evolves in the same way with age for the three languages, since there are no significant effects with the visual prompt*age group interaction $[F(4,114)=.935, p=.447]$.

\subsubsection{Incorrect pickups}

In order to evaluate the effectiveness of each language to locate a target without creating confusion with other objects in the game world, the number of times that children picked up the wrong object was counted. The data on incorrect pickups can be seen in Table 7 and in Figure 13 by visual prompt and age group. 


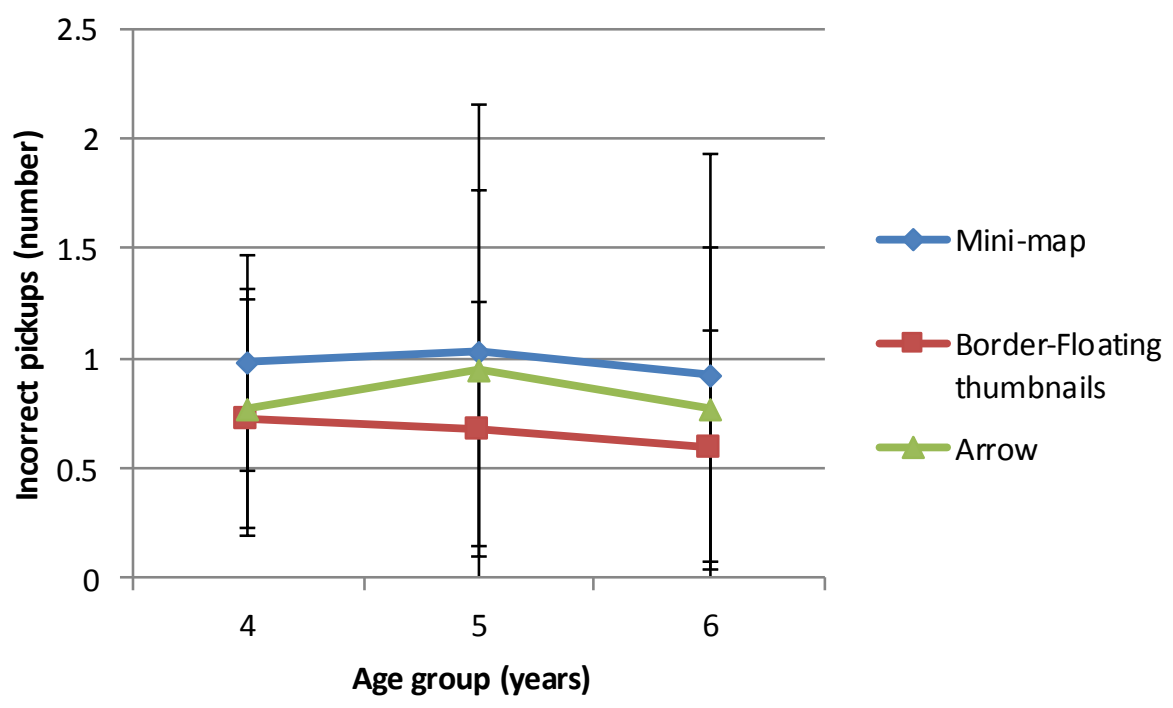

Figure 13. Number of incorrect pickups by visual prompt and age group.

The repeated measures ANOVA did not reveal significant differences in the within-subject factor visual prompt $[\mathrm{F}(1.750,99.774)=2.907, \mathrm{p}=.066]$ or in the between-subject factors Age group $[\mathrm{F}(2,57)=.367, \mathrm{p}=.694]$ and Gender $[\mathrm{F}(1,58)=.833, \mathrm{p}=.365]$.

The ANOVA also revealed that there are no significant differences in the evolution of the number of incorrect pickups with age for the three languages, since there are no significant effects with the visual prompt*age group interaction $[\mathrm{F}(3.501,99.774)=.144, \mathrm{p}=.952]$.

\section{Discussion}

The experiment explored and answered the comprehensive set of research questions that had been posed. The answer to RQ1 about whether there can be any feasible visual prompt to effectively provide directional awareness to kindergarten children is affirmative as the results reveal that children achieved success rates of over $90 \%$ in the evaluated languages, showing that the three evaluated directional awareness visual prompts can be understood by kindergarten children.

RQ2, on whether the effectiveness of the task is affected by the type of prompt, is answered affirmatively since the results show that significant differences were found between the three evaluated visual prompts. Despite having a success rate of $94 \%$, the mini-map technique was shown to cause children the most problems, whereas the border-floating thumbnails and the arrow techniques reached success rates of over $99 \%$. These results are interesting because they suggest that even very young children are able to perform the required mapping to interpret the data given by visual prompts and extrapolate it to locate different objects in a virtual world. The RQ2 for the Age factor (whether the effectiveness of the task is affected by age) is affirmatively answered, revealing that the youngest age group achieved a lower success rate than the older. As can be seen in Table 2, the above-mentioned issues with the mini-map happened exclusively 
with the youngest age group. As Piaget (Piaget 1973) points out, children begin their preoperational stage at 24 months and then gradually develop layers of symbolic behaviors as they are introduced to reading symbols (Gardner 1993), so the youngest children may have some issues when understanding the information communicated by the mini-map. This should be taken into account by designers when developing applications, because the use of the minimap technique with children aged 4 years or younger may have an impact on the effectiveness of the communication process.

RQ3, on whether the type of visual prompt has an impact on the efficiency of children in the task, is positively answered in terms of the time needed to complete the task, since the results showed that the mini-map technique was the slowest, followed by the border-floating thumbnails and finally the arrow technique was the fastest. Children have more difficulties and need more time to succeed in the task when the communication is given with the mini-map technique because mapping between what is being displayed on the mini-map and the virtual world is challenging for them and requires a mental context switch between both spaces. The next technique in time needed to succeed in the task is the border-floating thumbnails; in this case children have to understand that the thumbnail is being displayed on the intersection between the vector that links the main character to the target and the screen border and this mental demand may have an impact on the time. Finally, the arrow technique turned out to be the best in terms of completion time needed to succeed. In this case, the mental demand is lower since children "only" have to follow the direction to which the arrow points to reach the target. RQ3 is also affirmatively answered the Age factor, since the results show that there are differences between all the age groups, with the older children being faster. This is an expected result, given that children are continuously developing their motor and cognitive skills and the older they are the faster they are expected to perform the task. This should be taken into account by designers when time performance is a mandatory application requirement.

In response to RQ4, relative position awareness can be evaluated in several ways such as considering the optimality of the travelled path and the number of changes of direction. Analyzing the travelled path with respect to the visual prompt used, RQ4 is affirmatively answered. Although no differences in the distance of the travelled path are reported between the border-floating thumbnails and the arrow techniques, children travelled a significantly longer path when the mini-map was the communication technique used. As stated above, children may have some issues when making the mapping between what is being displayed on the mini-map to the virtual world and it has an impact on the travelled path, since they are not able to mentally define the appropriate path to follow at the beginning of the task. When performance in terms of the path is required in the application, designers should thus avoid using the mini-map technique. On the other hand, this research question is answered negatively for the Age factor, since age does not have an impact on the distance of the path covered. 
In response to RQ4 by means of another indirect measure, the number of changes of direction, the results show that overall, i.e. for all the techniques, the type of technique used does not have an impact on the number of changes of direction (see Figure 11). The answer to this research question is also negative for the Age factor, since it does not have an effect on the number of changes of direction made by the users. Therefore, there are no differences between techniques when communicating where the target is and the direction in which the character has to move to reach it.

In terms of visual interference with the task, we analyzed whether paying attention to the visual prompt used resulted in children accidentally picking up incorrect objects or colliding against blocks. When we analyze collisions with incorrect objects, RQ5 is answered negatively for the Visual prompt factor, since it does not affect the number of errors that children made when picking up objects. Age does not have an impact on the number of incorrect pickups made by users (RQ5 is negatively answered in terms of incorrect pickups for the Age factor). According to the data shown in Table 7, it can be seen that all the techniques have an average number of incorrect pickups lower than 1 by task, and so we can conclude that the techniques evaluated are effective in identifying the target and do not create ambiguity with the other objects scattered around the virtual world.

If we consider RQ5 in terms of the number of collisions with blocks in the task, then RQ5 is answered affirmatively for the Visual prompt factor. This is an interesting result since it shows that the evaluated techniques require different attention levels. The arrow technique was shown to be the best to reduce the number of collisions with the blocks. This can be explained because the arrow technique is shown around the main character and children do not have to look away to receive the directional awareness. However, with the other two techniques the visual cue is displayed at the bottom-right corner (mini-map) or the limits of the screen (border-floating thumbnails) forcing children to divert their attention from the main character and causing more collisions. The results also show differences between the mini-map and the border-floating thumbnails techniques, the first one being more distracting for children than the second. With the border-floating thumbnails, the required movement of the main character will always be "following" the thumbnail while it is moving across the screen, hence reducing the level of interference that diverts children's attention. However, when the mini-map technique is used, the focus is always on the bottom-right corner of the screen where the mini-map is placed. Hence, in situations in which the character has to move in the opposite direction, there is a continuous gaze context switch causing the maximum level of interference with the task. When the visual prompt's level of interference needs to be minimum, designers should use the arrow technique. RQ5 is also affirmatively answered for the Age factor since the older the children the 
fewer the number of collisions. This is an expected result since the older the children are the better they manage the cognitive load caused by visual context switch.

Finally, regarding the impact of gender, the results show that in general there are no differences between males and females in any of the evaluated dependent variables, so that all the research questions (RQ2, RQ3, RQ4 and RQ5) are answered negatively for the Gender factor. This is an interesting result since it shows that even though previous studies with pre-kindergarten children have found that, on average, preschool boys are more accurate than girls in spatial tasks and suggests that males develop visual-spatial cognition abilities before females (Levine et al. 1999), these possible development differences do not affect children in any aspect (effectiveness, efficiency, precision) when using the evaluated directional awareness visual prompts.

\section{Threats to validity}

Certain precautions should be taken before extrapolating the results obtained in this study to other contexts. Regarding the information on block locations, the arrow and the thumbnail mechanisms do not indicate where the blocks are placed, so the directional awareness mechanisms point to the target regardless of whether there is a block in the path or not. However, the mini-map mode included a small map containing all the elements in the virtual world and the blocks can be seen even though they are off the screen. This could have an impact, since the mini-map technique offers more information and could allow more precise paths. However, despite giving more information, the mini-map technique got the worst results in all the evaluated dimensions. On the other hand, the extra information offered by the minimap technique may have an impact on the time needed by children to complete the task, since they have to process more information. This limitation should therefore be taken into account when extrapolating the results.

In terms of measuring the changes of direction, each time the children change the direction button while piloting the spacecraft was counted as a change of direction. When they tried to move the character in a diagonal direction (switching between the horizontal and vertical arrows) these switches are counted as changes of direction. This could have some validity issues, since some children may want to drive the spacecraft in a diagonal path and the control interface does not allow it. However, the validity issue regarding the fact that children switch between the vertical and horizontal arrows to move the character diagonally will affect all the techniques in the same way, since the moving mechanism is the same for all the visual prompts. In future work it would be interesting to evaluate other indirect dragging techniques, such as a circular dial or a steering wheel to assess their suitability for use by kindergarten children.

Finally, it is worth noting that the American Academy of Pediatrics (AAP Council on communications and media, 2016) recommends avoiding the use of touch screen devices for 
children younger than 18 months and they also recommend a responsible usage with a limit of one hour of screen per day, with parents co-viewing and collaborating with the children's interactions when they are between two and five years old. It is also important to highlight that the use of touch technology cannot replace essential activities needed by young developing children, such as jumping, running, interacting with others, learning social interaction norms, etc.

\section{Conclusions}

In this work we analyzed a corpus of 100 commercial applications running on multi-touch devices for kindergarteners in order to determine how these applications use the virtual space. The analysis revealed that most of the evaluated applications are limited to simply using the screen size as the digital world that is always shown and only $33 \%$ of them used a space beyond the screen limits.There are few applications (only 5\% of the revised apps) that use some sort of prompt to provide directional awareness to users and there is no standard and validated way of providing this information. In order to assess kindergarteners' capacity to use the world beyond the screen boundaries and possible visual prompts to communicate directional spatial information, an experimental evaluation was carried out on children aged between 4 and 6 years old in a task requiring the use of a virtual space beyond the screen limits with the assistance of three different directional visual awareness prompts.

Our findings provide evidence that even though commercial applications do not use this augmented space, kindergarteners could complete a task requiring directional awareness with success rates of close to 100 per cent. Despite this type of skill being in the process of development, they are ready to interpret and extrapolate the data from the visual prompts to locate different objects in a virtual world.

The results revealed that the mini-map was the most problematic technique, whereas the borderfloating thumbnails and the arrow techniques reached success rates of over $99 \%$. The techniques evaluated were seen to have different performances in terms of the time needed to complete the task, the relative positioning awareness that they convey and the visual interference that they cause. In this respect, if the completion time is a mandatory requirement of the application, the arrow technique was the fastest, followed by the border-floating thumbnails and the mini-map technique was the slowest. On the other hand, if relative positioning awareness is one of the main requirements, designers should avoid using the mini-map technique, since it was significantly less effective than the arrow and the border-floating thumbnail techniques. Finally, when reducing the degree of interference of the visual prompts with the task is a priority, the most appropriate technique for this is the arrow language, followed by the border-floating thumbnail and the mini-map. This should be taken into account by designers when developing 
applications in order to choose the most appropriate technique to fit the applications requirements.

No differences were found in the effectiveness, efficiency or language understanding in terms of gender.

Our plans for future work include the design and evaluation of an educational scenario to study the impact of the proposed visual prompts in communicating multi-touch gestures (Nacher et al. 2017) and directional awareness in parents' dialogic strategies during the learning process. The goal will be to demonstrate that these visual prompts can improve the learning process by helping parents to focus on the learning content rather than on the children's expected interactions. We are also interested in studying whether the results obtained in this study can be extrapolated to a situation in which directional awareness is developed and communicated in a 3D space explored by kindergarteners with the use of digitally augmented tricycles (Tanaka \& Takahashi 2012) or collaborative robots (Garcia-Sanjuan et al. 2015).

\section{Acknowledgments}

This work received financial support from Spanish Ministry of Economy and Competitiveness and was funded by the European Development Regional Fund (EDRF-FEDER) in the project TIN2014-60077-R (SUPEREMOS). This work is also supported by a pre-doctoral fellowship within the FPU program from the Spanish Ministry of Education, Culture and Sports to V. Nacher (FPU14/00136) and from GVA (ACIF/2014/214) to F. Garcia-Sanjuan.

\section{References}

AAP Council on communications and media, 2016. Media and Young Minds. Pediatrics, 138(5), p.e20162591.

Abdi, H., 2010. The greenhouse-geisser correction. Encyclopedia of Research Design. Sage Publications, pp.544-548.

Baloian, N., Pino, J. a., Vargas, R., 2013. Tablet gestures as a motivating factor for learning. Proceedings of the 2013 Chilean Conference on Human - Computer Interaction ChileCHI '13, pp.98-103.

Basili, V.R., Caldiera, G., Rombach, H.D., 1994. The Goal Question Metric Approach, in: Encyclopedia of Software Engineering. Wiley, pp. 528-532.

Bradbury, D., 1978. The science of semiotics. New Literary History, 9(2), pp.199-204.

Bruner, J.S., 1960. The Act of Discovery. Philosophy of Education, p.137.

Case, R., Griffin, S., Kelly, W., 2001. Socio-economic differences in children's early cognitive 
development and their readiness for schooling. Psychological perspectives on early childhood education: Reframing dilemmas in research and practice, pp.36-63.

Case, R., Stephenson, K.M., Bleiker, C., Okamoto, Y., 2008. V. CENTRAL SPATIAL STRUCTURES AND THEIR DEVELOPMENT. Monographs of the Society for Research in Child Development, 61(1-2), pp.103-130.

Catling, S., 2005. Developing children's understanding and use of maps, in: Lee, C. and Hung, C. C. (eds) (Ed.), Primary Social Studies: Exploring Pedagogy and Content. Marshall Cavendish Education., Singapore.

Collins, A., Stevens, A.L., 1981. Goals and Strategies of Inquiry Teachers, Advances i. Hillsdale, N.J.: Lawrence Erlbaum Associates.

Cristia, A., Seidl, A., 2015. Parental Reports on Touch Screen Use in Early Childhood. PLOS ONE, 10(6), p.e0128338.

Demetriou, A., Christou, C., Spanoudis, G., Platsidou, M., 2002. The Development of Mental Processing: Efficiency, Working Memory, and Thinking. Monographs of the Society for Research in Child Development, 67(1), pp.1-167.

Dempsey, J. V., Rasmussen, K., Lucassen, B., 1994. Instructional gaming: Implications for instructional technology. Annual Meeting of the Association for Educational Communications and Technology, pp.1-21.

Derboven, J., De Roeck, D., Verstraete, M., 2012. Semiotic analysis of multi-touch interface design: The MuTable case study. International Journal of Human-Computer Studies, 70(10), pp.714-728.

Downs, R.M., Liben, L.S., Daggs, D.G., 1988. On Education and Geographers: The Role of Cognitive Developmental Theory in Geographic Education. Annals of the Association of American Geographers, 78(4), pp.680-700.

Falloon, G., 2013. Young students using iPads: App design and content influences on their learning pathways. Computers and Education, 68, pp.505-521.

Fernández-López, Á., Rodríguez-Fórtiz, M.J., Rodríguez-Almendros, M.L., Martínez-Segura, M.J., 2013. Mobile learning technology based on iOS devices to support students with special education needs. Computers \& Education, 61, pp.77-90.

Furió, D., González-Gancedo, S., Juan, M.C., Seguí, I., Rando, N., 2013. Evaluation of learning outcomes using an educational iPhone game vs. traditional game. Computers and Education, 64, pp.1-23.

Garcia-Sanjuan, F., Jaen, J., Nacher, V., Catala, A., 2015. Design and Evaluation of a Tangible Mediated Robot for Kindergarten Instruction, in: 12th International Conference on Advances in Computer Entertainment Technology. Iskandar, Malaysia.

Gardner, H., 1993. The Unschooled Mind. Fontana Press, London, UK.

Golbeck, S.L., 2005. Building Foundations for Spatial Literacy in Early Childhood. Proquest Journals, 60(6), p.72.

Goria, S., Papadopoulou, M., 2012. Icons versus symbols: investigating preschoolers' 
cartographic design., 5, pp.1-18.

Hiniker, A., Sobel, K., Hong, S.R., Suh, H., Irish, I., Kim, D., Kientz, J. a., 2015. Touchscreen Prompts for Preschoolers: Designing Developmentally Appropriate Techniques for Teaching Young Children to Perform Gestures. Proceedings of the 14th International Conference on Interaction Design and Children, pp.109-118.

Honomichl, R.D., Chen, Z., 2012. The role of guidance in children's discovery learning. Wiley Interdisciplinary Reviews: Cognitive Science, 3(6), pp.615-622.

Hourcade, J.P., 2007. Interaction Design and Children. Foundations and Trends® in HumanComputer Interaction, 1(4), pp.277-392.

Ioannou, A., Zaphiris, P., Loizides, F., Vasiliou, C., 2013. Let'S Talk About Technology for Peace: A Systematic Assessment of Problem-Based Group Collaboration Around an Interactive Tabletop. Interacting with Computers, p.iwt061-.

Jokisch, M., Bartoschek, T., Schwering, A., 2011. Usability testing of the interaction of novices with a multi-touch-table in semi public space, in: 14th international conference on Humancomputer interaction: interaction techniques and environments (HCII'11). Springer, pp. $71-80$.

Kähkönen, M., Ovaska, S., 2006. Initial observations on children and online instructions. Proceeding of the 2006 conference on Interaction design and children - IDC '06, pp.9396.

Leekam, S., Perner, J., Healey, L., Sewell, C., 2008. False signs and the non-specificity of theory of mind: Evidence that preschoolers have general difficulties in understanding representations. British Journal of Developmental Psychology, 26(4), pp.485-497.

Levine, S.C., Huttenlocher, J., Taylor, A., Langrock, A., 1999. Early sex differences in spatial skill. Developmental Psychology, 35(4), pp.940-949.

Malone, T., 1981. Toward a theory of intrinsically motivating instruction. Cognitive Science, 5(4), pp.333-369.

Markopoulos, P., Bekker, M., 2003. Interaction design and children. Interacting with Computers, 15(2 SPEC.), pp.141-149.

Mauchly, J.W., 1940. Significance Test for Sphericity of a Normal n-Variate Distribution. The Annals of Mathematical Statistics, 11(2), pp.204-209.

McKnight, L., Fitton, D., 2010. Touch-screen technology for children: Giving the Right Instructions and Getting the Right Responses, in: Proceedings of the 9th International Conference on Interaction Design and Children - IDC '10. ACM Press, New York, New York, USA, p. 238.

Van Der Meij, H., Van Der Meij, J., 2014. A comparison of paper-based and video tutorials for software learning. Computers and Education, 78, pp.150-159.

Mihajlov, M., Law, E.L.-C., Springett, M., 2014. Intuitive Learnability of Touch Gestures for Technology-Naive Older Adults. Interacting with Computers, 27(3), pp.344-356.

Nacher, V., Ferreira, A., Jaen, J., Garcia-Sanjuan, F., 2016. Are Kindergarten Children Ready for 
Indirect Drag Interactions?, in: Proceedings of the 2016 ACM on Interactive Surfaces and Spaces - ISS '16. ACM Press, New York, New York, USA, pp. 95-101.

Nacher, V., Garcia-Sanjuan, F., Jaen, J., 2016. Interactive technologies for preschool gamebased instruction: Experiences and future challenges. Entertainment Computing, 17, pp.19-29.

Nacher, V., Jaen, J., Catala, A., 2017. Evaluating Multitouch Semiotics to Empower Prekindergarten Instruction with Interactive Surfaces. Interacting with Computers, 29(2), pp.97-116.

Nacher, V., Jaen, J., Catala, A., 2014. Exploring Visual Cues for Intuitive Communicability of Touch Gestures to Pre-kindergarten Children, in: Proceedings of the Ninth ACM International Conference on Interactive Tabletops and Surfaces - ITS '14. ACM Press, New York, New York, USA, pp. 159-162.

Nacher, V., Jaen, J., Catala, A., Navarro, E., Gonzalez, P., 2014. Improving Pre-Kindergarten Touch Performance, in: Proceedings of the 9th ACM International Conference on Interactive Tabletops and Surfaces. ITS '14. ACM, New York, pp. 163-166.

Nacher, V., Jaen, J., Navarro, E., Catala, A., González, P., 2015. Multi-touch gestures for prekindergarten children. International Journal of Human-Computer Studies, 73, pp.37-51.

Neumann, M.M., 2017. Parent scaffolding of young children's use of touch screen tablets. Early Child Development and Care, pp.1-11.

Niemi, H., Ovaska, S., 2007. Designing spoken instructions with preschool children. Proceedings of the 6th international conference on Interaction design and children - IDC '07, p.133.

Peirce, C.S., 1902. Logic as semiotic: The theory of signs. Philosophical writings of Peirce, p. 100 .

Piaget, J., 1973. The Child and Reality. Grossman, New York.

Plowman, L., Stevenson, O., Stephen, C., McPake, J., 2012. Preschool children's learning with technology at home. Computers \& Education, 59(1), pp.30-37.

Rideout, V., 2011. Zero to Eight: Children's Media Use in America. Common Sense Media.

Smith, S.P., Burd, E., Rick, J., 2012. Developing, evaluating and deploying multi-touch systems. International Journal of Human-Computer Studies, 70(10), pp.653-656.

Tanaka, F., Takahashi, T., 2012. A tricycle-style teleoperational interface that remotely controls a robot for classroom children, in: Proceedings of the 7th annual ACM/IEEE international conference on Human-Robot Interaction. HRI '12.pp. 255-256.

Thomas, G. V., Nye, R., Robinson, E.J., 1994. How children view pictures: Children's responses to pictures as things in themselves and as representations of something else. Cognitive Development, 9(2), pp.141-164.

Vatavu, R., Cramariuc, G., Schipor, D.M., 2015. Touch interaction for children aged 3 to 6 years : Experimental findings and relationship to motor skills. International Journal of Human-Computer Studies, 74, pp.54-76. 


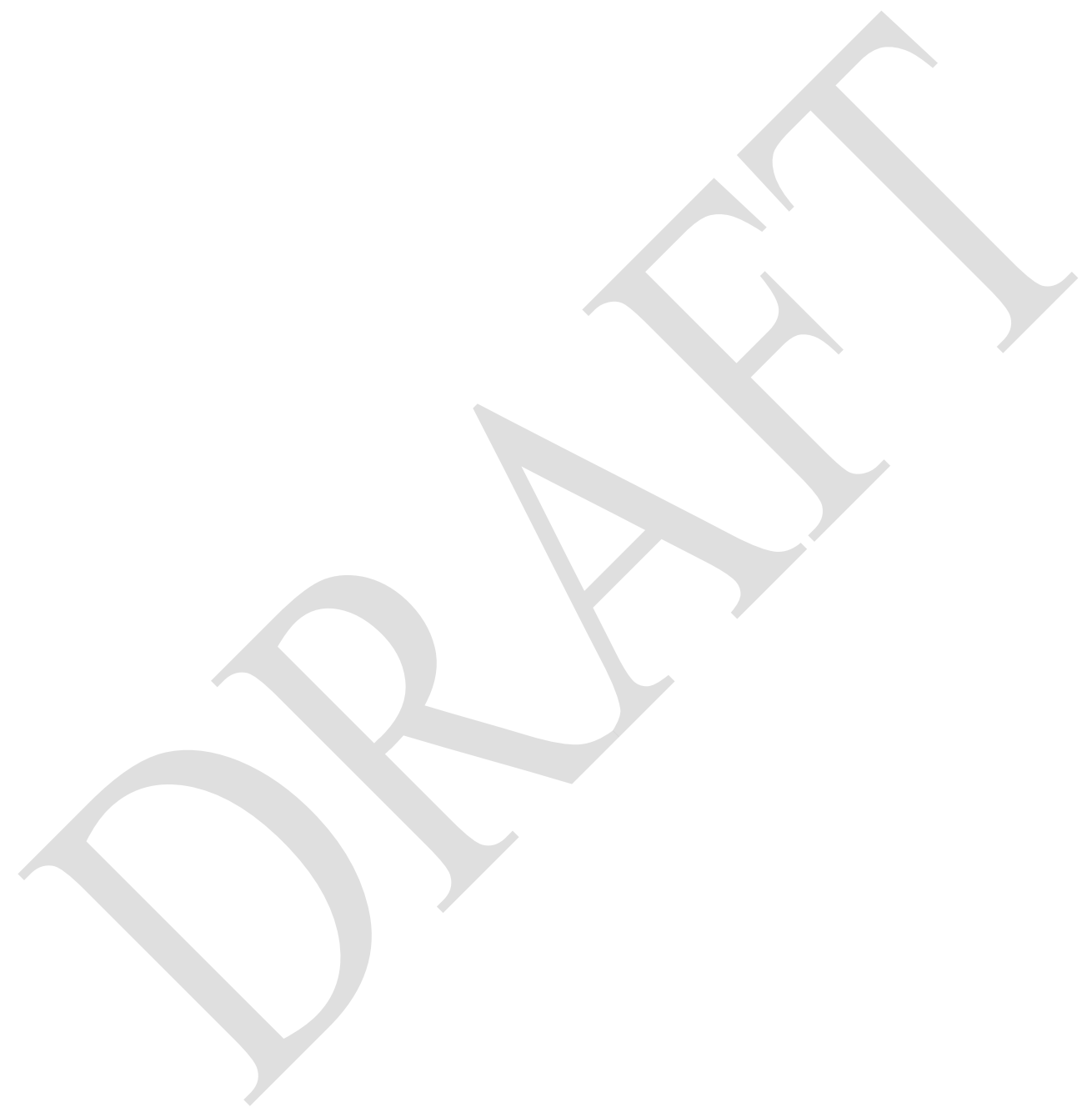




\section{Appendix}

Table 2. Success rate by visual prompt and age group.

\begin{tabular}{l|l|r|r}
\hline Visual prompt & Age Group & Average & SD \\
\hline \multirow{4}{*}{ Mini-map } & 4 & 86.11 & 29.04 \\
\cline { 2 - 4 } & 5 & 98.89 & 4.97 \\
\cline { 2 - 5 } & 6 & 97.78 & 9.94 \\
\cline { 2 - 5 } & Overall & 94.26 & 18.58 \\
\hline \multirow{2}{*}{ Border-Floating } & 4 & 100 & 0 \\
\cline { 2 - 5 } & 5 & 100 & 0 \\
\cline { 2 - 5 } & 6 & 99.44 & 2.48 \\
\cline { 2 - 5 } & Overall & 99.81 & 1.43 \\
\hline \multirow{5}{*}{ Arrow } & 4 & 99.44 & 2.48 \\
\cline { 2 - 5 } & 5 & 100 & 0 \\
\cline { 2 - 5 } & 6 & 100 & 0 \\
\cline { 2 - 5 } & Overall & 99.81 & 1.43 \\
\hline
\end{tabular}

Table 3. Completion time by visual prompt and age group (s).

\begin{tabular}{l|l|r|r}
\hline Visual prompt & Age Group & Average & SD \\
\hline \multirow{4}{*}{ Mini-map } & 4 & 415.42 & 78.71 \\
\cline { 2 - 4 } & 5 & 367.44 & 97.94 \\
\cline { 2 - 4 } & 6 & 335.46 & 47.53 \\
\cline { 2 - 4 } & Overall & 368.67 & 82.30 \\
\hline \multirow{2}{*}{ Border-Floating } & 4 & 342.64 & 62.63 \\
\cline { 2 - 5 } & 5 & 304.24 & 51.57 \\
\cline { 2 - 5 } & 6 & 268.00 & 44.55 \\
\cline { 2 - 5 } & Overall & 301.34 & 59.46 \\
\hline \multirow{5}{*}{ Arrow } & 4 & 336.04 & 47.86 \\
\cline { 2 - 5 } & 5 & 313.64 & 66.50 \\
\cline { 2 - 5 } & 6 & 262.79 & 33.00 \\
\cline { 2 - 5 } & Overall & 301.09 & 58.90 \\
\hline
\end{tabular}

Table 4. Percentage of increment in the travelled path in comparison with the ideal.

\begin{tabular}{l|l|r|r}
\hline Visual prompt & Age Group & Average & SD \\
\hline \multirow{4}{*}{ Mini-map } & 4 & 27.31 & 16.06 \\
\cline { 2 - 4 } & 5 & 28.09 & 19.81 \\
\cline { 2 - 5 } & 6 & 27.01 & 15.56 \\
\cline { 2 - 5 } & Overall & 27.48 & 17.03 \\
\hline \multirow{2}{*}{ Border-Floating } & 4 & 25.54 & 16.61 \\
\cline { 2 - 5 } thumbnails & 5 & 22.50 & 21.04 \\
\cline { 2 - 5 } & 6 & 16.45 & 13.23 \\
\cline { 2 - 5 } & Overall & 21.37 & 17.46 \\
\hline \multirow{4}{*}{ Arrow } & 4 & 19.38 & 11.22 \\
\cline { 2 - 5 } & 5 & 22.02 & 15.64 \\
\cline { 2 - 5 } & 6 & 14.63 & 9.23 \\
\cline { 2 - 5 } & Overall & 18.71 & 12.6 \\
\hline
\end{tabular}


Table 5. Changes of direction by visual prompt and age group.

\begin{tabular}{l|l|r|r}
\hline Visual prompt & Age Group & Average & SD \\
\hline \multirow{4}{*}{ Mini-map } & 4 & 16.27 & 4.91 \\
\cline { 2 - 4 } & 5 & 17.85 & 5.83 \\
\cline { 2 - 4 } & 6 & 18.62 & 5.34 \\
\cline { 2 - 4 } & Overall & 17.58 & 5.37 \\
\hline \multirow{2}{*}{ Border-Floating } & 4 & 16.85 & 4.29 \\
\cline { 2 - 5 } & 5 & 17.37 & 2.94 \\
\cline { 2 - 5 } & 6 & 16.48 & 1.64 \\
\cline { 2 - 5 } & Overall & 16.90 & 3.11 \\
\hline \multirow{4}{*}{ Arrow } & 4 & 16.8 & 3.32 \\
\cline { 2 - 5 } & 5 & 18.12 & 4.67 \\
\cline { 2 - 5 } & 6 & 17.13 & 2.74 \\
\cline { 2 - 5 } & Overall & 17.35 & 3.65 \\
\hline
\end{tabular}

Table 6. Number of collisions with blocks by visual prompt and age group.

\begin{tabular}{l|l|r|r}
\hline Visual prompt & Age Group & Average & SD \\
\hline \multirow{4}{*}{ Mini-map } & 4 & 1.88 & 1.31 \\
\cline { 2 - 4 } & 5 & 1.41 & 1.43 \\
\cline { 2 - 5 } & 6 & 0.97 & 1.04 \\
\cline { 2 - 5 } & Overall & 1.88 & 1.31 \\
\hline \multirow{2}{*}{ Border-Floating } & 4 & 1.75 & 1.63 \\
\cline { 2 - 5 } thumbnails & 5 & 1.75 & 1.58 \\
\cline { 2 - 5 } & 6 & 0.73 & 0.72 \\
\cline { 2 - 5 } & Overall & 1.41 & 1.44 \\
\hline \multirow{5}{*}{ Arrow } & 4 & 1.2 & 0.91 \\
\cline { 2 - 5 } & 5 & 1.08 & 1.35 \\
\cline { 2 - 5 } & 6 & 0.62 & 0.68 \\
\cline { 2 - 5 } & Overall & .97 & 1.04 \\
\hline
\end{tabular}

Table 7. Number of incorrect pickups by visual prompt and age.

\begin{tabular}{l|l|r|r}
\hline Visual prompt & Age Group & Average & SD \\
\hline \multirow{4}{*}{ Mini-map } & 4 & .98 & .49 \\
\cline { 2 - 5 } & 5 & 1.03 & 1.12 \\
\cline { 2 - 5 } & 6 & .92 & 1.01 \\
\cline { 2 - 5 } & Overall & .98 & .90 \\
\hline \multirow{3}{*}{ Border-Floating } & 4 & .73 & .54 \\
\cline { 2 - 5 } thumbnails & 5 & .68 & .58 \\
\cline { 2 - 5 } & 6 & .60 & .53 \\
\cline { 2 - 5 } & Overall & .67 & .54 \\
\hline \multirow{5}{*}{ Arrow } & 4 & .77 & .54 \\
\cline { 2 - 5 } & 5 & .95 & .81 \\
\cline { 2 - 5 } & 6 & .77 & .73 \\
\cline { 2 - 5 } & Overall & .83 & .70 \\
\hline
\end{tabular}


Table 8. P-value of the post-hoc pair-wise comparison of visual prompt for all the dependent variables.

\begin{tabular}{l|r|r|r}
\hline \multicolumn{1}{c|}{ Visual prompts } & $\begin{array}{c}\text { Mini-map vs } \\
\text { Border-Floating } \\
\text { thumbnails }\end{array}$ & $\begin{array}{c}\text { Mini-map vs } \\
\text { Arrow }\end{array}$ & $\begin{array}{c}\text { Border-Floating } \\
\text { thumbnails vs } \\
\text { Arrow }\end{array}$ \\
\hline Success & .061 & .064 & 1.000 \\
\hline Completion time & $<.001$ & $<.001$ & 1.000 \\
\hline Travelled path & .150 & $\mathbf{. 0 0 3}$ & .745 \\
\hline Changes of direction & 1.000 & 1.000 & 1.000 \\
\hline Collision with obstacles & .041 & $<.001$ & .400 \\
\hline Incorrect pickups & .129 & .497 & .628 \\
\hline
\end{tabular}




\section{Curriculum Vitae}

Vicente Nacher, MSc (2013, Universitat Politécnica de València - UPV) has been granted a fellowship from the Ministry of Education to work with the FutureLab team at the Software Engineering and Information Systems (ISSI) research group. He obtained the Best Undergraduate Student Award in Computer Science at the UPV and the first prize in the National End-ofCareer Awards by the Ministry of Education in 2012. His current research

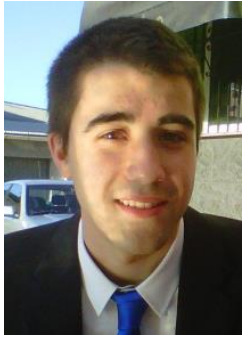
interests include multi-touch usability and collaborative systems for children.

Sandra Jurdi, MSc (2016, Universitat Politécnica de València), B Eng (2015, Universitat Jaume I) is a contract researcher and member of the Futurelab team within the Software Engineering and Information Systems research group. Her current research interests include Tangible User Interfaces, Gameful Collaborative Problem Solving, and technological social support systems for pediatric patients.

Javier Jaen, PhD (2006, Universitat Politécnica de Valencia), MSc (1998, Virginia Tech), DEA (1994, INSA de Lyon) is currently an associate professor with the Laboratory of Advanced Information Systems at the Department of Computing and Information Systems. His current research interests include ubiquitous computing, ambient intelligence and tabletop-based computing. He

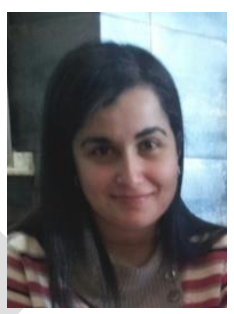
was the recipient of a Fulbright scholarship and is a member of the Upsilon Pi Epsilon International Honor Society for Computing and Information Disciplines. Dr. Jaen was the winner of the Best PhD Dissertation in Computer Science Award and the Social Council's Award at the UPV, and won the eMobility Award in 2003.

Fernando Garcia-Sanjuan, MSc (2013, Universitat Politécnica de València), Eng (2012 - UPV) is a PhD candidate at UPV and member of the Futurelab team within the Software Engineering and Information Systems research group. His current research interests include Tangible User Interfaces, MultiDisplay Environments, and Computer-Supported Collaborative Learning.

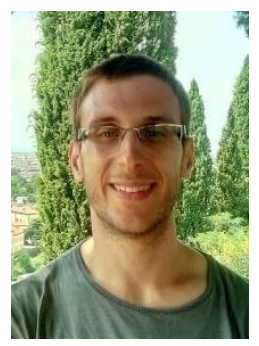

